\title{
Susceptibility of Human Oral Squamous Carcinoma Cells (OSCC) H103 and H376 cell lines to Retroviral OSKM mediated reprogramming
}

Nalini Devi Verusingam ${ }^{1}$ ， Swee Keong Yeap ${ }^{2}$, Huynh Ky ${ }^{3}$ ， Ian C Paterson ${ }^{4}$ ，Suan Phaik Khoo ${ }^{5}$, Soon Keng Cheong ${ }^{1,6}$, Alan HK Ong ${ }^{\text {Corresp., }}{ }^{1}$, Tunku Kamarul ${ }^{7}$

${ }^{1}$ Faculty of Medicine and Health Sciences, Universiti Tunku Abdul Rahman, Selangor, Malaysia

2 Institute of Bioscience, Universiti Putra Malaysia, Selangor, Malaysia

3 College of Agriculture and Applied Science, Cantho University, Vietnam

4 Department of Oral Biology \& Biomedical Sciences, Faculty of Dentistry, Universiti Malaya, Kuala Lumpur, Malaysia

5 School of Dentistry, International Medical University, Kuala Lumpur, Malaysia

6 MAKNA Cancer Research Institute, Kuala Lumpur, Malaysia

7 Tissue Engineering Group, National Orthopaedic Centre of Excellence for Research and Learning, Department of Orthopaedic Surgery, Faculty of Medicine, University Malaya, Kuala Lumpur, Malaysia

Corresponding Author: Alan HK Ong

Email address: onghk@utar.edu.my

Although numbers of cancer cell lines have been shown to be successfully reprogrammed into induced pluripotent stem cells (iPSCs), reprogramming Oral Squamous Cell Carcinoma (OSCC) to pluripotency in relation to its cancer cell type and the expression pattern of pluripotent genes under later passage remain unexplored. In our study, we reprogrammed and characterised $\mathrm{H} 103$ and $\mathrm{H} 376$ oral squamous carcinoma cells using retroviral OSKM mediated method. Reprogrammed cells were characterized for their embryonic stem cells (ESCs) like morphology, pluripotent gene expression via quantitative real-time polymerase chain reaction (RT-qPCR), and immunofluorescence staining, embryoid bodies (EB) formation and directed differentiation capacity. Reprogrammed H103 (Rep-H103) exhibited similar ESCs morphologies with flatten cells and clear borders on feeder layer. Reprogrammed H376 (Rep-H376) did not show ESCs morphologies but grow with a disorganized morphology. Critical pluripotency genes Oct4, Sox2 and Nanog were expressed higher in Rep-H103 against the parental counterpart from passage 5 to passage 10. As for Rep-H376, Nanog expression against its parental counterpart showed a significant decrease at passage 5 and although increased in passage 10, the level of expression was similar to the parental cells. Rep-H103 exhibited pluripotent signals (Oct4, Sox2, Nanog and Tra-1-60) and could form EB with the presence of three germ layers markers. Rep-H103 displayed differentiation capacity into adipocytes and osteocytes. The OSCC cell line $\mathrm{H} 103$ which was able to be reprogrammed into an iPSC like state showed high expression of Oct4, Sox2 and Nanog at late passage and may provide a potential iPSC 
model to study multi-stage oncogenesis in OSCC. 


\section{Susceptibility of Human Oral Squamous Carcinoma Cells (OSCC) H103 and H376 cell lines to Retroviral OSKM mediated reprogramming}

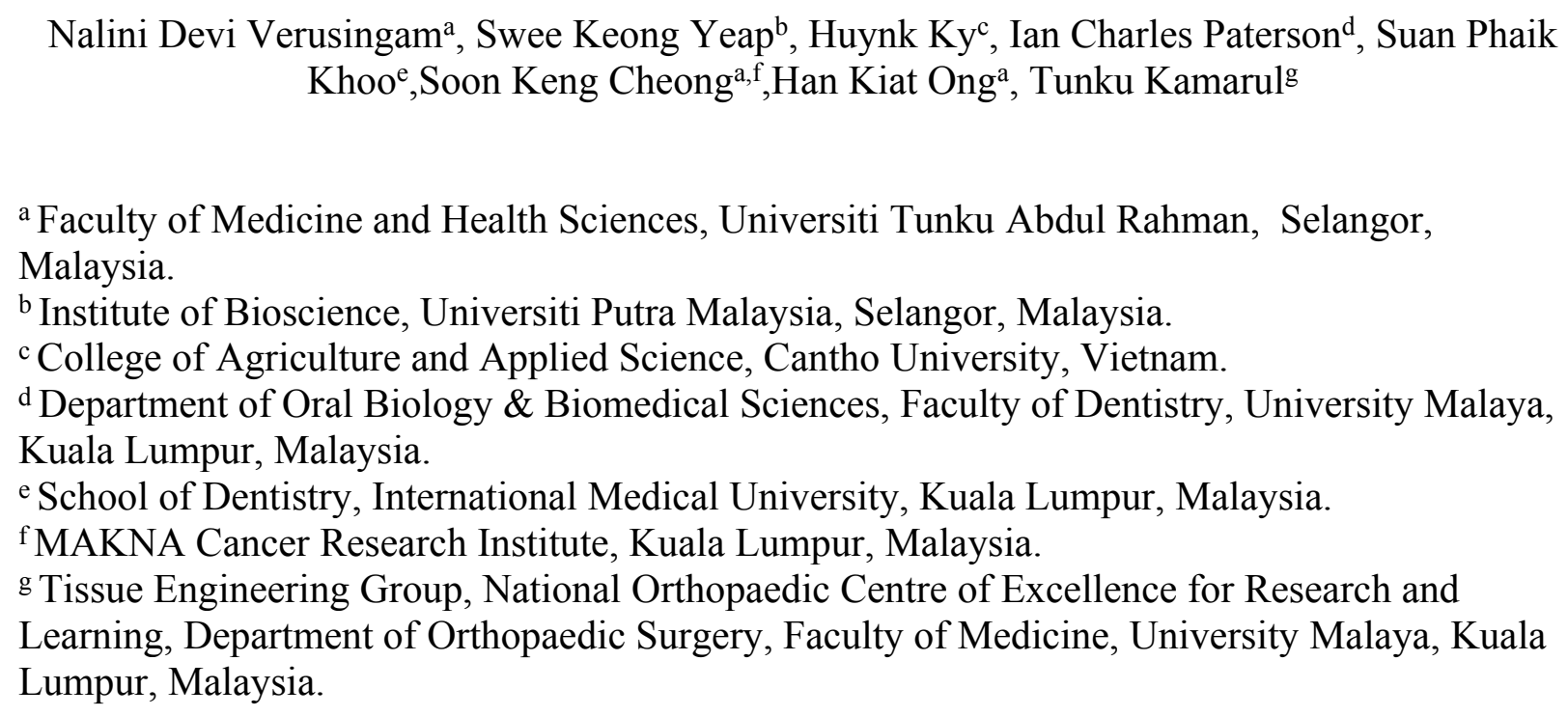
Kuala Lumpur, Malaysia.

${ }^{\mathrm{e}}$ School of Dentistry, International Medical University, Kuala Lumpur, Malaysia.

${ }^{\mathrm{f}}$ MAKNA Cancer Research Institute, Kuala Lumpur, Malaysia.

g Tissue Engineering Group, National Orthopaedic Centre of Excellence for Research and Learning, Department of Orthopaedic Surgery, Faculty of Medicine, University Malaya, Kuala Lumpur, Malaysia.

*Corresponding author at Universiti Tunku Abdul Rahman, Malaysia

Email address: onghk@utar.edu.my 
37

38

39

40

41

42

\section{Introduction}

Oral cancer is the sixth most common malignancy worldwide and is more prevalent in the developing than developed countries (Warnakulasuriya, 2009). More than $90 \%$ of oral cancer cases are classified as Oral Squamous Cell Carcinoma (OSCC) which is a malignant epithelial cancer that arises from oral keratinocytes (Scully \& Bagan, 2009). As there are no reliable diagnostic markers and early stage OSCC is asymptomatic, patients with OSCC are often presented for treatment with advanced stage cancer resulting in a poor prognosis. (Warnakulasuriya, 2009; Markopoulos, Michailidou \& Tzimagiorgis, 2010)

In spite of the advancement in molecular based detection of cancer stages (Bose et al., 2012; Rhandawa \& Archaraya, 2015), the underlying biological mechanisms that take place within the OSCC progression have not been well established. Existing models for OSCC study derived from xenograft of primary tumours have been problematic as in-vitro studies have resulted in low percentage of cell number and induced mutations with prolonged culture. Furthermore, human cell models of OSCC from tumour cell lines generally can only mimic the advanced tumour state which does not allow the different stages of cancer to be monitored and studied (Shirako et al., 2015). Hence, these unresolved issues necessitate an approach to establish human cancer models that recapitulate OSCC progression.

57 Originally, induced pluripotent stem cells (iPSCs) were established from adult human somatic stem cells through the transient expression of Yamanaka'sOct4, Sox2, KLf4 and c-Myc (OSKM) transcription factors reprogramming technology (Takahashi \&Yamanaka., 2007)and used as a promising stem cell source to overcome ethical and immune rejection issues that often 
61 surfaced when using human Embryonic Stem Cells (hESCs) (Yamanaka, 2007).Subsequent

62 studies demonstrated that reprogrammed cancer cells were able to exhibit pluripotent capacity

63 and differentiation tendency, which were distinct from that of their parental cells with much of

64 the observable changes owing to epigenetic effects of reprogramming(Miyoshi et al.,

65 2009; Carette et al., 2010;Mahalingam et al., 2012; Gandre-babbe et al., 2013; Kim et al., 2013;

66 Zhang et al., 2013; Choong et al. 2014;Koga et al., 2014; Kotini et al., 2015; Lee et al.,

67 2015).Interestingly, while the majority of the reprogrammed cancer cells showed either lower or

68 a loss of tumorigenicity, Carette et al., 2010\& Gandre-babbe et al.,2013 indicated a re-

69 establishment of the oncogenic dominance over the pluripotent cell phenotype when the reprogrammed cell types were differentiated into hematopoietic lineage cells and Kim and

71 colleagues, 2013 showed that the reprogrammed human pancreatic cancer cells were able

72 recapitulate its cancer progression from early to late stage of cancer development upon

73 differentiation. In contrast, MCF-7 breast cancer cell line which was reprogrammed via

74 retroviral-OSKM method, failed to show distinct pluripotent signals and was unable to

75 differentiate into 3 primary germ layers but displayed instead, a typical cancer stem cell (CSC)

76 phenotype (Corominas-Faja et al., 2013). These reprogrammed phenotypes of cancer cells have

77 made the study of cancer development and underlying cellular systems more feasible, much of

78 which were not previously encountered from any available cancer models especially in

79 addressing cancer progression, discovery of cancer specific biomarkers and more effective

80 therapy (Lang, Shi \& Chin, 2013;Lee et al., 2015;Kim \& Zaret, 2015).

To the best of our knowledge, no studies have previously reported on the reprogramming

of OSCC cancer cells and therefore in the present study, we examined the susceptibility of two 
84 OSCC cell lines to be reprogrammed into pluripotency and their respective pluripotent gene 85 expression patterns.

\section{Materials and Methods}

\section{Cell Culture} Malaysia. Cell lines were cultured in DMEM/F12 supplemented with $10 \%$ fetal bovine serum (FBS) (Gibco/Invitrogen, USA), Hydrocortisone $(0.5 \mathrm{ug} / \mathrm{ml})$ (Sigma-Aldrich, USA) at $37^{\circ} \mathrm{C}$ in the presence of 5\% CO2. Reprogrammed OSCC cells were maintained in conditioned hESC medium consisting DMEM/F12 (Gibco/Invitrogen, USA), supplemented with 20\% knock-out serum replacement (Gibco/Invitrogen, USA), $0.1 \mathrm{mM}$ nonessential amino acids (Gibco/Invitrogen, USA), $4 \mathrm{mM}$ L-glutamine (Sigma-Aldrich, USA), $10 \mathrm{ng} / \mathrm{mL}$ fibroblast growth factor (bFGF) (Invitrogen, USA) and 0.1 mM 2-mecaptoethanol (Sigma-Aldrich, USA). The medium was changed every 48 hours.

Retrovirus Production and Infection

Retroviral vectors were produced via packaging cell lines, 293FT cell lines (human embryonal kidney cells) (Thermo Fisher Scientific, USA) using the Yamanaka Factors - (Oct4, Sox2. Klf4, c-Myc). Vectors, pMX-based retroviral hOct4 (Plasmid 17217) (Addgene, USA), hSox2 (Plasmid 17218) (Addgene, USA), hKlf4 (Plasmid 17219) (Addgene, USA), hc-Myc 
107 (Plasmid 17220) (Addgene, USA), retroviral gag-pol packaging plasmid (Plasmid 8449)

108 (Addgene, USA), VSV-G expression plasmid (Plasmid 8454) (Addgene, USA) and pMX-GFP

109 (Cell Biolabs, USA) used in this experiments were provided by Dr. Shigeki Sugii, DUKE-NUS

110 Graduate Medical School, Singapore. Human embryonal kidney cell lines were plated at $3.6 \mathrm{x}$

$11110^{6}(70 \%-80 \%$ confluency) one day before transduction. The packaging cell lines were then

112 transfected with Retro-GFP/OSKM vectors along with the transfection reagent

113 LIPOFECTAMINE 2000 (Life Technologies, USA) according to the manufacturer's protocol.

114 The supernatant was collected at 48hours of post-transfection and filtered with PVDF filtered

115 (45uM) (Merck Milipore, USA). Polybrene $(0.5 \mathrm{ul} / \mathrm{ml}$ from $10 \mathrm{mg} / \mathrm{ml})$ was then added into the

116 supernatant containing Retroviral Vectors to enhance the transduction efficiency within the

117 target cell types. Presence of selection marker, green fluorescent protein (GFP) in H103 and

118 H376 were counted manually using the cell count option in ImageJ program.

Retroviral Infection and iPSCs Cell Generation

Human Oral Squamous Carcinoma Cells (H103 and H376) were seeded at $7.5 \mathrm{x}$

$12210^{4}$ cells/well on 6 well plates, 24 hours before transduction. Equal amounts of supernatant cell lines. Infected cells were incubated overnight. The medium was changed at 24 hours of postspinfection at1 hour and 30 minutes $\left(32^{\circ} \mathrm{C}\right)$ before placing the plates into a hypoxic incubator $127\left(5 \% \mathrm{O}_{2}\right)$. At day 3 post infection, OSCC cell lines were harvested by trypsinization and re-plated with 1 x $10^{4}$ cells/well on 6 well plates containing irradiated Mouse Embryonic Feeder Layer 129 (MEF) (GlobalStem, USA). After 24 hours, the medium was replaced with hESCs medium. 
130 Fifteen days after transduction, formed colonies were picked up and transferred onto a new

131 mouse embryonic fibroblast (MEF) feeder layer (GlobalStem, USA). These colonies were

132 propagated at least up to five passages before being subjected to pluripotency characterization.

133

134

135

136

137

138

139

140

141

142

143

144

145

146

147

148

149

150

151

152

Gene Expression Assessment in OSCC-Induced Pluripotent Stem Cells (iPSCs)

Ribonucleic acid (RNA) was extracted from parental H103 and H376 and respective reprogrammed counterparts using RNeasy Mini Kit (Qiagen, Germany) according to the manufacturer's protocols. Total RNA was then converted into complementary deoxyribonucleic acid (cDNA) via reverse transcription (QuantiTech Reverse Transcriptase Kit). The fluorescent signals from each sample were plotted against cycle numbers which represented the accumulation of product over the duration of the quantitative real-time PCR (qRT-PCR) experiment. Quantitative real-time PCR amplification efficiency generated with primers (Supplementary Table 1) were optimized using hESC, a commonly used gold standard for pluripotency characterization. Beta-actin (ACTB) was used as a house keeping gene in this experiment. Briefly, the condition used in this experiment comprises initial denaturation at $95^{\circ} \mathrm{C}$ for 15 minutes, followed by 40 cycles of denaturation at $94^{\circ} \mathrm{C}$ for 30 seconds with annealing at $60^{\circ} \mathrm{C}$ for 30 seconds and extension at $72^{\circ} \mathrm{C}$. Following qRT-PCR, fluorescence data collection was performed during extension. Real-time PCR was performed with iQ5 Bio-Rad qPCR machine (Bio-Rad, USA) using Quantitect Sybr Green PCR Master Mix (Qiagen, Germany). iQ5 Optical System Software, Version 2.0 was used for the analysis. 
153

154

155

156

157

158

159

160

161

162

163

164

165

166

167

168

169

170

171

172

173

174 Stained cells were observed under Zeiss Imager A.1 Fluorescence Microscope (Carl Zeiss, 175 Germany).

\section{Immunofluorescense Staining}

Cells were fixed using 4\% v/v paraformaldehyde (Sigma-Aldrich, USA), washed three times with PBS containing 1\% BSA and permeablized using Perm Buffer (BD Biosciences, USA) for 15 minutes at room temperature (Intracellular markers). After permeabilization, cells were blocked with PBS containing 1\% BSA for 1 hour at room temperature after the blocking solution was removed and cells were washed three times with PBS. Cells were then incubated with conjugated antibodies in PBS containing $1 \%$ BSA overnight at $4{ }^{\circ} \mathrm{C}$. Antibodies used were at 1:50 dilution factor for Oct4-PE (BD Biosciences, USA), Sox2-PE (BD Biosciences, USA) Nanog-ALexa Fluor 488 (BD Biosciences, USA), and Tra-1-60-PE (BD Biosciences, USA). After the overnight incubation, cells were washed three times with PBS and stained with DAPI ANTIFADE GOLD (Invitrogen, USA) prior to viewing under Zeiss Imager A.1 Fluorescence Microscope (Carl Zeiss, Germany).

\section{Embryoid Bodies (EB's) Formation}

Rep-H103 cells were seeded onto ultra-low attachment plates (Corning) containing commercialize embryoid bodies medium (Milipore, USA). Transferred cells were grown in suspension for 8 days. The medium was consistently changed every $2-3$ days up to 8 days without disrupting the EBs. EBs formed were carefully collected for immunofluorescence staining to determine the presence of three germ layers specific markers (Ectoderm: OTX2, Sox1; Endoderm: Sox17, Gata4; Mesoderm: Brachyury) according to the manufacturer's instructions (Human Three Germ Layer 3-Color Immunostainining Kit) (R\&D Systems, USA). 
177 Directed Differentiation of Human H103 iPSCs like Cells

178 Since H103 cell line is of the ectoderm and endoderm lineage (Jones \& Klein, 2013),

179 reprogrammed H103 was subjected to directed differentiated into adipocytes and osteocytes

180 which is of the mesoderm lineages in order to access its differentiation potential.

181

182

183

184

185

186

187

188

189

190

191

192

193

194

195

196

197

198

Osteogenic Assay

Osteogenic differentiation medium (ODM) (Erenpreisa \& Cragg, 2013) was used to induce mineralization or osteogenesis in reprogrammed H103. IPSC-like cells were plated on gelatine coated six-well plates and cultured in 3ml of ODM consisting $90 \%$ DMEM/F12 (Gibco/Invitrogen, USA), 10\% FBS (Gibco/Invitrogen, USA) supplemented with 10nM dexamethasone (Sigma-Aldrich, USA), 20mM $\beta$-glycerol phosphate (Sigma-Aldrich, USA), and $50 \mu \mathrm{M}$ L-ascorbic acid (Sigma-Aldrich, USA). The medium was changed every 2-3 days. After 21 days of incubation at $37^{\circ} \mathrm{C}, 5 \% \mathrm{CO}_{2}$, cells were stained in Alizarin Red for visualization of calcium deposits. Stained cells were then evaluated under Eclipse TS100 inverted microscope (Nikon, Japan) and images were captured for analysis. Presence of mineralized osteoblasts indicated bright orange-red precipitate.

\section{Adipogenic Assay}

Colonies plated on gelatine coated six-well plates and were cultured in $3 \mathrm{ml}$ of adipogenic differentiation medium (ADM) (Erenpreisa \& Cragg, 2013)per well. ADM consist of 90\% DMEM/F12 (Gibco/Invitrogen, USA), 10\% FBS (Gibco/Invitrogen, USA) supplemented with $0.5 \mu \mathrm{M}$ dexamethasone, $0.5 \mu \mathrm{M}$ isobuthylmethylxanthine and $50 \mu \mathrm{M}$ indometacin. The medium 
199 was changed every 2-3 days. After 21 days of incubation at $37^{\circ} \mathrm{C}, 5 \% \mathrm{CO}_{2}$, cells were stained in

200 Oil Red O for visualization of lipid droplets. Stained cells were then viewed under Eclipse 201 TS100 inverted microscope (Nikon, Japan) and images were captured for analysis. Mature 202 adipocytes containing intracellular lipid vesicles were stained bright red.

203

204

205

206

207

208

209

210

211

212

213

214

215

216

217

218

219

220

221

Statistical Analysis

Statistical data analysis was carried out with Paired t-Tests to compare the quantitative outcome of parental, reprogrammed counterparts at passage 5 and reprogrammed counterparts at passage 10 of H103 and H376 cell lines using SPSS, Software version 22.0. All tests were conducted at $95 \%$ confidence level and all data were presented as mean \pm standard error of mean SEM. The differences were considered significant at $\mathrm{P}<0.05$.

\section{Results}

Transfection efficiencies in oral squamous Cells carcinoma cell lines

Green Fluorescence Protein (GFP) serves as an internal control and used for evaluating transduction efficiency. Uptake of vector pMX-GFP was tested using the highly transfectable human embryonic kidney cells (293FT) (Supplementary Figure 1).Subsequently, transduction efficiencies were examined in OSCC cell lines using vector pMX-GFP (16.5 $\mu \mathrm{g})$ which encodes for green fluoresecent protein signals to confirm the uptake of transgenes prior to OSKM transduction. GFP signals were detected in $\mathrm{H} 103$ and $\mathrm{H} 376$ at 48 hours confirming the uptake of transgenes (Fig 1A). The transduction efficiency of $75.50 \% \pm 1.52$ and $38.20 \% \pm 0.60$ obtained from H376 and H103 cells respectively showed an almost 2-fold increase in H376(Fig 1B). Negative control was performed to access the potential influence of reprogramming on the viability of the cells (Supplementary Figure 2). 
223

224

225

226

227

228

229

230

231

232

233

234

235

236

237

238

239

240

241

242

243

244

Reprogramming of oral squamous cell carcinoma cell lines

Clones were picked approximately two weeks after transduction (Fig. 2A) in both H103 and H376 cells. Distinct morphological patterns and changes were observed between the parental cancer cells and their reprogrammed counterparts (Fig. 2B i-iii \& Fig 2Ci-iv). Only Rep-H103 clones were able to be passaged above passage 5 (Fig. 2B-iv) while clones derived from RepH376 differentiated at passage 2 onwards and were unable to sustain iPS-like cell morphology (Fig. 2C-v). Although higher GFP transfection capacity was achieved in H376, stable clones were successfully generated only from H103 cell line as these clones were able to be expanded up to passage 20 and still maintained an ESC-like morphology (Fig. 2B iv-v). Morphologies of all derived clones from $\mathrm{H} 103$ are highly distinct from its parental cells with clear borders on mouse embryonic fibroblast feeder layer, high nucleus to cytoplasmratio and the colonies displayed small cells morphology with spaces between them (Fig. 2B iv-v).

\section{Differential Gene Expression}

Overall, the expressions of endogenous Oct4, Sox2 in Rep-H103 cells were much higher than that of Rep-H376. However, suppression of Klf4 and c-Myc was seen in both reprogrammed cells (Fig. 3). Pluripotent marker expression levels of the reprogrammed cells relative to the parental counterparts which were quantified using qRT-PCR (Fig. 3A) showed down-regulation of Oct4 expression in rep-H103 at passage 5 but increased with a 2.80 fold change at passage 10. Expression of Sox2 (Fig. 3B) showed gradual up-regulation in Rep-H103 at passage 5 and passage 10 with 55 and77 fold change respectively. Oncogenic Klf4 (Fig 3C) gene expression in reprogrammed H103 was significantly down regulated upon reprogramming 
245 and the level of expression maintained throughout passage 5 and passage 10. Similar gene

246 expression pattern as Klf4 was observed in c-Myc gene (Fig 3D) but at a much lower expression

247 level. Nanog expression was also up-regulated gradually across passage 5 with 2.84 fold change

248 to passage 10 with 7.07 fold change relatively to its parental counterpart (Fig 3E).

249

In the case of Rep-H376, Oct4 expression showed an initial reduction at passage 5 of-

4.35 fold change but was up-regulated at passage 10 with 1.07 fold change (Fig 3A). Gradual increase of Sox2expression (Fig 3B) from passage 5 to passage 10 was observed in Rep-H376, a similar pattern to that of Rep-H103. Down-regulation of Klf4 expression was observed at passage 5 but slightly increased from -3.13 fold change to -1.89 respectively at passage 10 (Fig at passage 10 (Fig 3D). Nanog expression was found reduced in Rep-H376 at passage 5 with 1.79 fold change and showed little increase at passage 10 with 1.86 fold change (Fig. 3E).

Rep-H103 continuously proliferated as adherent, flat colonies under feeder conditions and captured satisfactory pluripotent signals from real-time PCR analysis compared to reprogrammed H376. Therefore, successfully Rep-H103 cells were characterised further for its pluripotency protein expression via immunofluorescence analysis. Rep-H103 expressed the pluripotency markers of Oct4, Sox2, Nanog and Tra-1-60 indicating a distinct difference between H103 derived iPS-like cells (Fig. 4A)over its parental cancer cell line (Fig. 4B). 

week in EB specific medium. At day 8, EBs were then evaluated for the presence of three germ layers via immunofluorescence staining. EBs was also induced from parental cell line of H103 to serve as control (Fig. 5A-i). Rep-H103 showed morphologically compact round borders EB (Fig. 5A-ii). As, H103 cell line was derived from both ectodermal and endodermal lineage expressions signals from these two lineages were expected to be detected in EBs from both the parental and the reprogrammed counterpart(Fig. 5B-i and 5B-ii). However, mesoderm (Fig. 5Biii) expression was only detected in EBs derived from Rep-H103 indicating cross lineage differentiation ability of the rep-H103 upon reprogramming into an IPS-like cell.

Directed Differentiation Assay

Crystal formation was observed during the osteogenesis process whereby the volume of the matrix mineralization increased during the 21 days of incubation as indicated from the Alizarin Red S stained calcium deposits (Fig. 6A-ii). In addition, tiny vesicles containing lipid droplets had formed in the cytoplasm of the cells after 21 days of incubation. The accumulation of lipid droplets was stained positive with Oil Red O staining (Fig. 6A-iv).

\section{Discussion}

Overall, different responses towards retroviral-OSKM mediated reprogramming were observed in two different grade OSCC cell lines (H103-STNMP Stage I and H376-STNMP 
290 line and generated H103 iPS-like cells could undergo in-vitro expansion more than 20 passages.

291 The ESC-like morphological changes in Rep-H103 corresponded to other reprogrammed cancer

292 cell lines including chronic myeloid leukemia (CML) cancer cell line (KBM7 cells) (Carette et

293 al., 2010), lung cancer cells (Mahalingam et al., 2012), liver cancer cells (Zhang et al.,

294 2014)melanoma cells (Lin et al., 2008) and osteosarcoma (Zhang et al., 2013; Choong et al.,

295 2014). On the other hand, reprogrammed H376 tended to differentiate into its original phenotype

296 and the ESC-like features were no longer observable after passage 2 (Fig. 2C-v).

Although Rep-H103 cell had successfully acquired pluripotent ectopic expressions and sustained its pluripotency potentiality, it possessed lower GFP transgene uptake efficiency as compared to that of Rep-H376. (Fig. 1).This feature is consistent with a previous reported study

300 in which, reprogramming of four different osteosarcoma cell lines (Saos-2, MG-63, U-2 OS and G-292) via Retroviral-OSKM mediated system demonstrated that U-2 OS cell line which was highly responsive to GFP transduction, eventually lost its pluripotency capacity upon reprogramming and could not be maintained in prolonged in-vitro culture. Presence of various intrinsic factors between the osteosarcoma cancer cell lines was hypothesized to contribute to multiple responses towards reprogramming (Choong et al., 2014).As such, depending on the cell type, uptake of OSKM transgenes may not necessarily be correlated with transduction efficiency.

Reprogramming resistance was observed in H376 cell line in which the expressions of 308 key pluripotent genes were insignificant and the ESC-like morphological features eventually diminishes with subsequent passage. Reprogramming roadblocks contributed by diverse 310 molecular properties and biophysical nature of the cell type could result in inefficient 311 reprogramming (Vierbuchen \& Wernig, 2012). Notably, the tumour suppressor gene, p53 which

312 safeguards the cellular genome integrity was shown to lower reprogramming efficiency and 
313 kinetics by removing DNA damaged cells at the early stages of the reprogramming stepwise

314 process via apoptosis (Vierbuchen \& Wernig, 2012; Spike \& Wahl, 2011). Although both H103

315 and H376 harbours the mutant p53 gene, H376 carries a p53 gene; a nonsense mutation and

316 expresses the truncated form of the protein which basically do not show any detectable mutant

317 p53 expression (Yeudal et al., 1995). Despite the fact that the mutated form of p53 gene may

318 provide a more favourable condition for reprogramming cancer cells (Ebrahimi, 2015;

319 Vierbuchen \& Wernig, 2012; Spike \& Wahl, 2011), the presence rather than the absence of the

320 mutant p53 expression has been reported to enhance reprogramming efficiency (Sarig et al.,

321 2010; Tapia \& Scholer, 2010) as in the case of H103 which was able to maintain its pluripotent

322 features under prolonged passage. Furthermore, it has been shown that TGF- $\beta$ triggers

323 epithelial-mesenchymal transition (EMT) (Ebrahimi, 2015) and the presence of transforming

324 growth factor beta-I (TGF- $\beta$ ) signal transduction was previously reported to be among the root

325 causes of roadblocks in reprogramming( $\mathrm{Li}$ et al., 2010). Moreover, successful reprogramming

326 towards pluripotency was shown to be facilitated by mesenchymal-to-epithelial transition (MET)

327 followed by suppression of epithelial-to mesenchymal transition (EMT) regulation (Chen, Han \&

328 Pei, 2012). Paterson and colleagues determined the effect of transforming growth factor beta-I

329 (TGF- $\beta$ ) in OSCC H-series cell lines in which H376 cell line was shown to be more responsive

330 to TGF- $\beta$ than H103 (Paterson et al., 1995). As such, H376 may potentially harbour higher EMT

331 activity which makes it more resistance towards reprogramming.

332 It was previously showed that extensive passaging enhances the reprogramming process

333 (Chin M. H., et al, 2009) in which the hierarchical pluripotency gene activation takes place in a

334 gradual manner upon prolong in-vitro passaging (Shan Z.Y., et al, 2014). Notably, in our study,

335 down-regulation of Oct4 in P5 for rep-H103may indicate that its expression was not fully 
336 activated at the initial phase at P5 but its expression was more distinct at a later phase at P10

337 whereby more stable expression of pluripotent genes are expected to occur within the

338 reprogramming process. Nevertheless, the fundamental pluripotency regulators (Oct4, Sox2 and

339 Nanog) in Rep-H103 were expressed at higher levels than that of Rep-H376 at both passage 5

340 and passage 10. We observed that Nanog expression indicated a distinct difference between the

341 two cell types and their susceptibility towards reprogramming. The interactions between Oct4,

342 Sox2 and Nanog had been demonstrated via mutagenesis in-vitro assay and in-vivo functional

343 study described previously in which suppression of Oct4 and Sox2 expression respectively

344 decreases the promoter activity of Nanog (Rodda et al., 2005). Furthermore, the deficiency in

345 Nanog expression results in partially reprogrammed cells which are unable to shift into

346 pluripotency state due to impaired regulation of pluripotency network (Festuccia et al., 2013).

347 Such was the case as reported by Miyoshi et al., (2009) in which gastrointestinal cell lines

348 selected for reprogramming were shown to express low level of Nanog mRNA, but gradually

349 acquired significant up-regulation of Nanog expression upon reprogramming with four

350 pluripotent transcription factors. In our studies, further evidence of pluripotency was confirmed

351 on successfully Rep-H103 via the immunofluorescence staining for common intracellular (Oct4,

352 Sox2, Nanog) and extracellular (Tra-1-60) pluripotent markers, which were used in pluripotent

353 stem cells characterization. All intracellular and extracellular pluripotent markers were detected

354 on reprogrammed H103 indicating pluripotency expressions were achieved at the protein level.

355 As Klf4 is known to act either as a tumour suppressor gene or an oncogene, depending on

356 the need of the tumour cells and the types of cancer (Evans \& Liu, 2008) and c-Myc is a crucial

357 oncogene that confers immortality in cancer cells via a shift from senescence state to oncogenic

358 progression (Erenpreisa \& Cragg, 2013), these transcription factors have been highly implicated 
359 in influencing cancer progression. The down-regulation pattern of oncogenic gene expression,

360 namely c-Myc and K1f4 in both H103 and H376 were also-observed in reprogrammed human

361 osteosarcoma cells (Zhang et al., 2013). Furthermore, when teratoma formations were assessed,

362 it was found that the parental cancer cells formed tumour at a faster rate than that of the

363 reprogrammed counterpart which exhibited a reduced aggressive cancer phenotype. Down-

364 regulation of both c-Myc and Klf4 in reprogrammed OSCC suggests reprogramming may initiate

365 an epigenetic reversal process on the oncogenic gene networks in cancer cells and this

366 phenomenon could be utilized as a therapeutic strategy for treatment of OSCC.

367 The differentiation potential demonstrated that Rep-H103 which initially originated from

368 ectoderm and endoderm lineage (Jones \& Klein, 2013), portrays its capability of differentiating

369 into osteocytes and adipocytes which are of the mesoderm lineage. The ability to differentiate

370 into all three germ layers has been commonly observed in successfully reprogrammed cells

371 including iPS-like cancer cells (Carette et al., 2010; Rizzino, 2013) and is crucial in generating

372 post-IPSCs to unravel the underlying tumorigenesis development of the specific cancer.

Reprogrammed OSCC cell lines (H103 and H376) were used as a cancer-specific model

to provide a conceptual study on the cells ability to be reprogrammed into a pluripotent state and

their pluripotent characteristics at the in-vitro level. Since our study involves a stage I (H103)

and stage III (H376) OSCC cell lines, the findings obtained does not address the effect of

377 reprogramming on stage IV OSCC (the final stage in the STNMP classification) and the

378 outcomes of the pluripotent signals at the in-vivo level. The next phase of the study is being 379 planned to address the current limitation of the findings.

In summary, the maintenance of stem cell like morphology and pluripotent expressions

upon reprogramming was only observed in rep-H103 cells. This may be due to the differences in 
382 the inherent genetic make-ups between H103 and H376 cell lines, which determined their 383 capacity to be reprogrammed into iPS-like cells. Our findings indicated a typical up-regulation 384 pattern of endogenous transcription factors - Oct4, Sox2 and Nanog in the reprogrammed H103 385 cell. However, down-regulation of oncogenes, c-Myc and Klf4 was observed in both Rep-H103

386

387

388

390

391

392

393

394

395

396

397

398

399

400

401

402

403

404

405

406

407

408

409

410 and Rep-376 cells, As such, the OSCC reprogrammed cells are potential models for further studies on cancer progression in OSCC, by enabling access to cancer properties from the initial tumour initiation to the later malignant/metastatic states as well as models for the discovery of novel anti-cancer drugs.

\section{Acknowledgments}

We would like take this opportunity to thank Assistant Professor Dr. Shigekii Sugii from DUKENUS Graduate Medical School, Singapore, for sharing his iPSCs knowledge and technology with us. We would also like to thank Dr. Teoh Hoon Koon, Teh Hui Xin and Choong Pei Fen who sequenced the plasmids.

\section{References}

Bose P, Klimowicz AC, Kornaga E, Petrillo SK, Matthews TW, Chandarana S, Magliocco AM, Brockton NT, Dort JC.2012. Bax expression measured by AQUA analysis is an independent prognostic marker in oral squamous cell carcinoma. BMC Cancer12(1):1.

Brana I, Siu LL.2012. Locally advanced head and neck squamous cell cancer: treatment choice based on risk factors and optimizing drug prescription.Annals of Oncology23(10):x178-x185.

Carette JE, Pruszak J, Varadarajan M, Blomen VA, Gokhale S, Camargo FD, Wernig M, Jaenisch R, Brummelkamp TR. 2010. Generation of iPSCs from cultured human malignant cells. Blood. 115(20):4039-42. 
Chen J, Han Q, Pei D.2012. EMT and MET as paradigms for cell fate switching. Journal of molecular cell biology4(2):66-69.

413 T.2014.Heterogeneity of osteosarcoma cell lines led to variable responses in reprogramming. International Journal of Medical Science 11:1154-60. of luminal-like breast cancer cells generates Sox2-overexpressing cancer stem-like cellular states harboring transcriptional activation of the mTOR pathway. Cell Cycle12(18):31093124.

Ebrahimi B.2015. Reprogramming barriers and enhancers: strategies to enhance the efficiency and kinetics of induced pluripotency. Cell Regeneration4(1):1.

Erenpreisa J, Cragg MS.2013. Three steps to the immortality of cancer cells: senescence, polyploidy and self-renewal. Cancer Cell Int3(1):92.

Evans P M, Liu C.2008. Roles of Krupel-like factor 4 in normal homeostasis, cancer and stem cells. Acta Biochim Biophys Sin (Shanghai)40(7):554-64.

Festuccia N, Osorno R, Wilson V, Chambers I.2013. The role of pluripotency gene regulatory network components in mediating transitions between pluripotent cell states. Current Opinion in Genetics \& Development 23(5):504-11.

Gandre-Babbe S, Paluru P, Aribeana C, Chou ST, Bresolin S, Lu L, Sullivan SK, Tasian SK, Weng J, Favre H, Choi JK.2013.Patient-derived induced pluripotent stem cells recapitulate hematopoietic abnormalities of juvenile myelomonocytic leukemia. Blood 121(24):4925-9.

Jones KB, Klein OD.2013. Oral epithelial stem cells in tissue maintenance and disease: the first steps in a long journey. International Journal of Oral Sciences5(3):121-9.

Kim J, Hoffman JP, Alpaugh RK, Rhim AD, Reichert M, Stanger BZ, Furth EE, Sepulveda AR, Yuan CX, Won KJ, Donahue G. 2013.An iPSC line from human pancreatic ductal adenocarcinoma undergoes early to invasive stages of pancreatic progression. Cell Reports3(6):2088-99.

452

Kim J, Zaret KS. Reprogramming of human cancer cells to pluripotency for models of cancer progression. 2015. The EMBO Journal34(6):739-47.

Koga C, Kobayashi S, Nagano H, Tomimaru Y, Hama N, Wada H, Kawamoto K, Eguchi H, Konno M, Ishii H, Umeshita K. 2014. Reprogramming using microRNA-302 
453

454

455

456

457

458

459

460

461

462

463

464

465

466

467

468

469

470

471

472

473

474

475

476

477

478

479

480

481

482

483

484

485

486

487

488

489

490

491

492

493

494

improves drug sensitivity in hepatocellular carcinoma cells. Annals of Surgical Oncology 21(4):591-600.

Kotini AG, Chang CJ, Boussaad I, Delrow JJ, Dolezal EK, Nagulapally AB, Perna F, Fishbein GA, Klimek VM, Hawkins RD, Huangfu D.2015. Functional analysis of a chromosomal deletion associated with myelodysplastic syndromes using isogenic human induced pluripotent stem cells. Nature biotechnology33(6):646-55.

Lang JY, Shi Y, Chin YE.2013. Reprogramming cancer cells: back to the future. Oncogene32(18):2247-8.

Lee DF, Su J, Kim HS, Chang B, Papatsenko D, Zhao R, Yuan Y, Gingold J, Xia W, Darr H, Mirzayans R. 2015.Modeling Familial Cancer with Induced Pluripotent Stem Cells. Cell161(2):240-54.

Lee SY, Park HR, Cho NH, Choi YP, Rha SY, Park SW, Kim SH.2013. Identifying genes related to radiation resistance in oral squamous cell carcinoma cell lines. International Journal of Oral and Maxillofacial Surgery42(2):169-76.

Li R, Liang J, Ni S, Zhou T, Qing X, Li H, He W, Chen J, Li F, Zhuang Q, Qin B.2010.A mesenchymal-to-epithelial transition initiates and is required for the nuclear reprogramming of mouse fibroblasts. Cell stem cell7(1):51-63.

Lin SL, Chang DC, Chang-Lin S, Lin CH, Wu DT, Chen DT, Ying SY.2008.Mir-302 reprograms human skin cancer cells into a pluripotent ES-cell-like state. RNA14(10):21152124.

López-Otín C, Blasco MA, Partridge L, Serrano M, Kroemer G.2013.The Hallmarks of Aging. Cell 153(6):1194-1217.

Mahalingam D, Kong CM, Lai J, Tay LL, Yang H, Wang X.2012. Reversal of aberrant cancer methylome and transcriptome upon direct reprogramming of lung cancer cells. Sci Rep 2:592.

Markopoulos AK, Michailidou EZ, Tzimagiorgis G. 201o. Salivary markers for oral cancer detection. The open dentistry journal27;4(1).

Miyoshi N, Ishii H, Nagai KI, Hoshino H, Mimori K, Tanaka F, Nagano H, Sekimoto M, Doki Y, Mori M.2009. Defined factors induce reprogramming of gastrointestinal cancer cells. PNAS107(1):40-5.

O-charoenrat P, Pillai G, Patel S, Fisher C, Archer D, Eccles S, Rhys-Evans P.2003.Tumour thickness predicts cervical nodal metastases and survival in early oral tongue cancer. Oral oncology39(4):386-390. 
Paterson IC, Patel V, Sandy JR, Prime SS, Yeudall WA.1995. Effects of transforming growth factor beta-1 on growth-regulatory genes in tumour-derived human oral keratinocytes. British journal of cancer72(4):922.

Petersen PE.2005. Strengthening the prevention of oral cancer: the WHO perspective. Community Dental Oral Epidemiology33(6):397-9.

Rhandawa V, Archaraya V.2015.Integrated network analysis and logistic regression modeling identify stage-specific genes in Oral Squamous Cell Carcinoma. BMC Medical Genomics8(1):39.

Rizzino A.2013. Concise Review: The Sox2-Oct4 Connection: Critical Players in a Much Larger Interdependent Network Integrated at Multiple Levels. Stem Cells31(6):1033-9.

Rodda DJ, Chew JL, Lim LH, Loh YH, Wang B, Ng HH, Robson P. 2005. Transcriptional Regulation of Nanog by OCT4 and SOX2. Journal of Biological Chemistry 280:24731-24737.

Sarig R, Rivlin N, Brosh R, Bornstein C, Kamer I, Ezra O, Molchadsky A, Goldfinger N, Brenner O, Rotter V.2010.Mutant p53 facilitates somatic cell reprogramming and augments the malignant potential of reprogrammed cells. The Journal of Experimental Medicine207(10):2127-2140.

Scully C, Bagan J.2009. Oral squamous cell carcinoma overview. Oral Oncology 45(45):301-8.

Shenoi R, Devrukhkar V, Sharma BK, Sapre SB, Chikhale A. 2012. Demographic and clinical profile of oral squamous cell carcinoma patients: A retrospective study. Indian journal of cancer49(1):21.

Shirako Y, Taya Y, Sato K, Chiba T, Imai K, Shimazu Y, Aoba T, Soeno Y.2015. Heterogeneous tumor stromal microenvironments of oral squamous cell carcinoma cells in tongue and nodal metastatic lesions in a xenograft mouse model. Journal of Oral Pathology of Medicine44(9):656-68.

Spike BT, Wahl GM.2011. P53, stem cells, and reprogramming tumor suppression beyond guarding the genome. Genes \& cancer2(4):404-19.

Takahashi K, Tanabe K, Ohnuki M, Narita M, Ichisaka T, Tomoda K, Yamanaka S.2007. Induction of pluripotent stem cells from adult human fibroblasts by defined factors. Cell131(5):861-72.

Tapia N, Schöler HR.2010. P53 connects tumorigenesis and reprogramming to pluripotency. The Journal of experimental medicine207(10):2045-2048. 
539

540

541

542

543

544

545

546

547

548

549

550

551

552

553

554

555

556

557

558

559

560

561

562

563 564

565

566

567

568

Vierbuchen T, Wernig M.2012. Molecular roadblocks for cellular reprogramming. Molecular cell47(6):827-838.

Warnakulasuriya S.2009."Global epidemiology of oral and oropharyngeal cancer."Oral oncology45(4):309-316.

Yamanaka, S. 2007. Strategies and new developments in the generation of patient-specific pluripotent stem cells. Cell Stem Cell. 1: 39-49.

Yeudall WA, Paterson IC, Patel V, Prime SS.1995. Presence of human papillomavirus sequences in tumour-derived human oral keratinocytes expressing mutant p53. European Journal of Cancer Part B: Oral Oncology 31(2):136-143.

Zhang DM, Li JJ, Yan P, Hu JT.2014.Establishment and identification of induced pluripotent stem cells in liver cancer patients. Asian Pac J Trop Med7(4):253-6.

Zhang P, Zhang Z, Zhou X, Qiu W, Chen F, Chen W.2006. Identification of genes associated with cisplatin resistance in human oral squamous cell carcinoma cell line. $B M C$ Cancer6(1):224.

Zhang X, Cruz FD, Terry M, Remotti F, Matushansky I.2013.Terminal differentiation and loss of tumorigenicity of human cancers via pluripotency-based reprogramming. Oncogene32(18):2249-60. 


\section{Figure 1}

Fig 1 - Transduction Efficiency of Retroviruses in OSCC

(A) Oral squamous carcinoma cell lines ( $\mathrm{H} 103$ and H376) were introduced with ecotropic pMXs retroviruses containing GFP cDNA. The upper panel shows the images of bright-field and fluorescent microscope, Zeiss Axiovert inverted microscope, original magnification: 20x. (B) The lower panel shows the percentages of cells transduced with GFP. Data are expressed as mean \pm standard deviation (SD).

$\mathbf{A}$

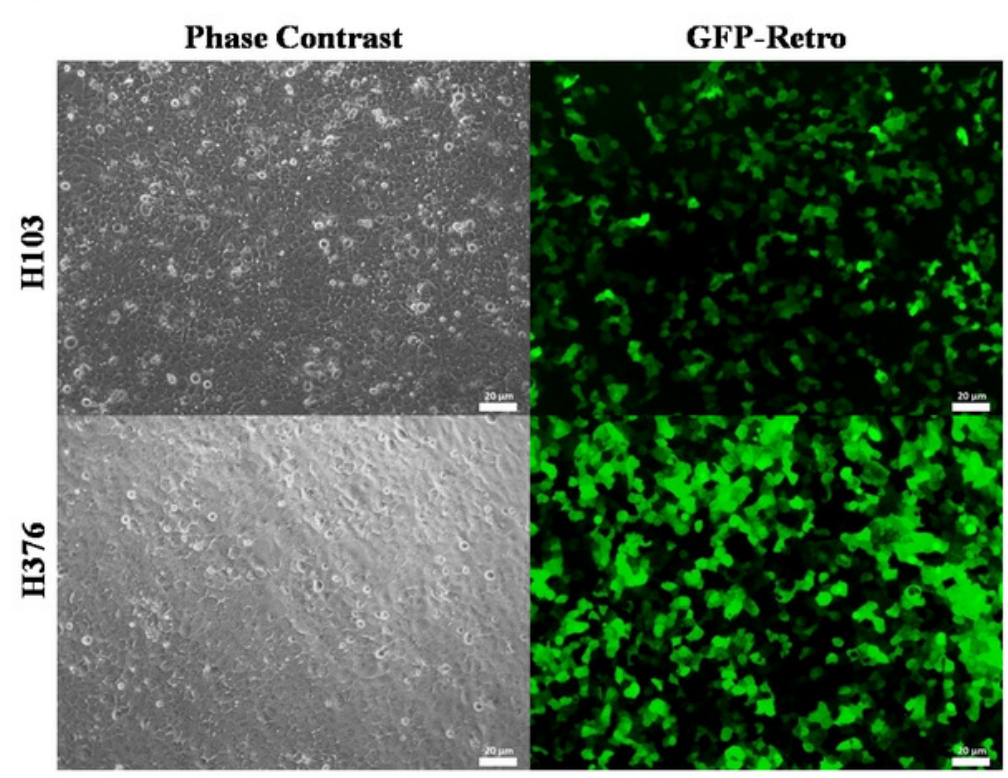

B

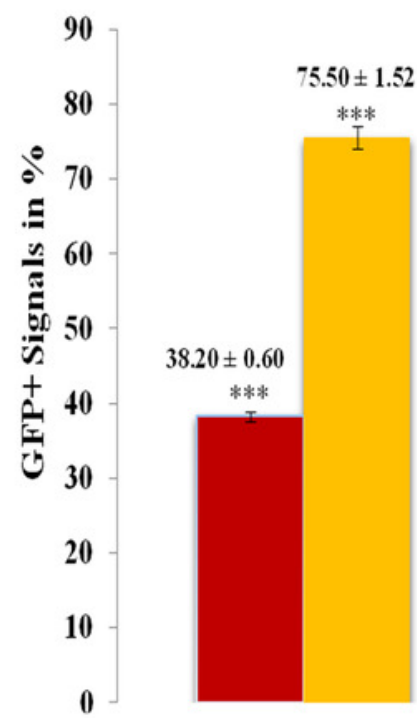

-H103 ( Grade I) H376(Grade III) 


\section{Figure 2}

Fig 2 - Induction of Pluripotent cells from OSCC

(A) Timeline of iPSCs induction. (B-i) Morphology of H103. (B-ii) Morphology of transduced

$\mathrm{H} 103$ at 24 hours post infection. (B-iii) Emergence of iPSCs like colonies from reprogrammed H103 on MEF feeder layer at day 15 (Red arrow). (B-iv-v) Images of established stable H103 iPSCs like cells at passage number 10. (C-i) Morphology of H376. (C-ii) Morphology of transduced H376 at 24 hours post infection. (C-iii) Emergence of IPSC like colonies from H376 on MEF feeder layer at day 15 (Red arrow). (C-vi) Image of iPSC like cells from H376 at P2. (C-v) Differentiated reprogrammed H376 (Red arrows), collected at P5 and P10 for real time expressions analysis.

A

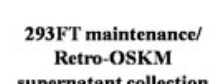

supernatant collection

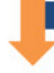

2 Weeks

B

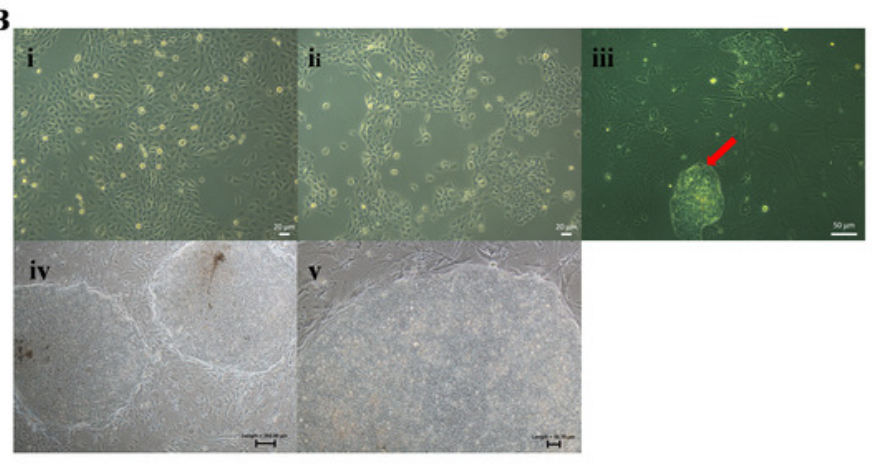

C

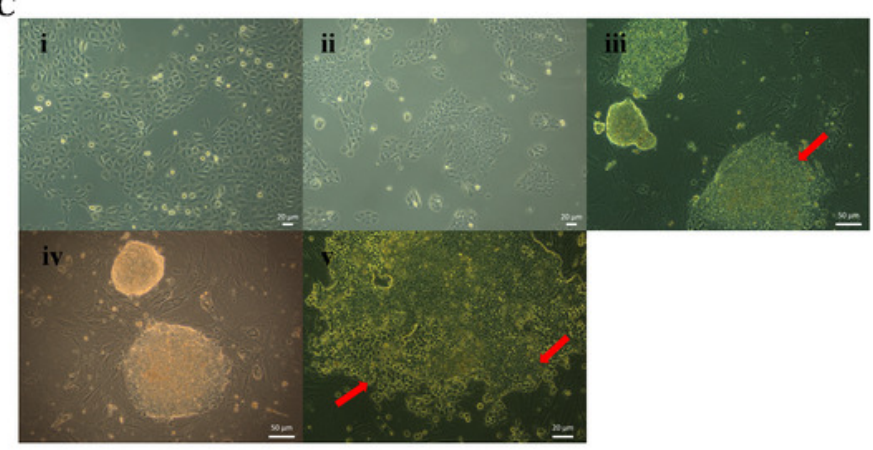


Figure 3

Fig 3 - In-Vitro mRNA expression of pluripotent genes in reprogrammed H103 and H376 Relative to Parental (n-3)

(A-E) Data presented as mean \pm SEM. Statistical differences are indicated with $*$ for $\mathrm{P}<0.05$, ** for $\mathrm{P}<0.01$ and $* * *$ for $\mathrm{P}<0.001$ using paired t-test.

$\mathbf{A}$

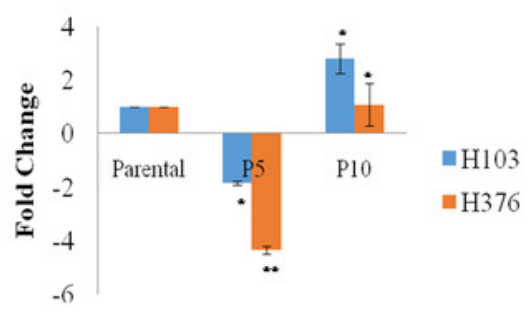

C

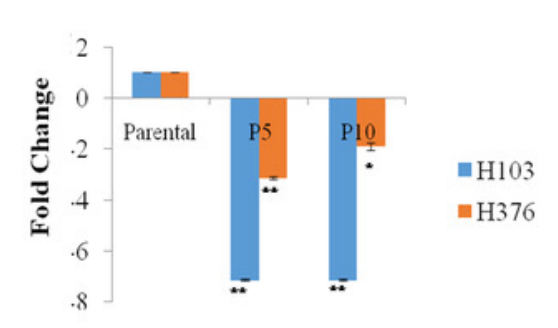

B

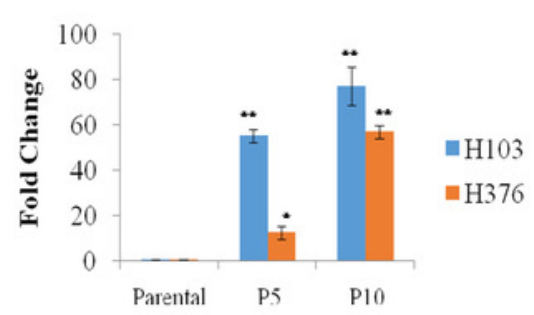

D

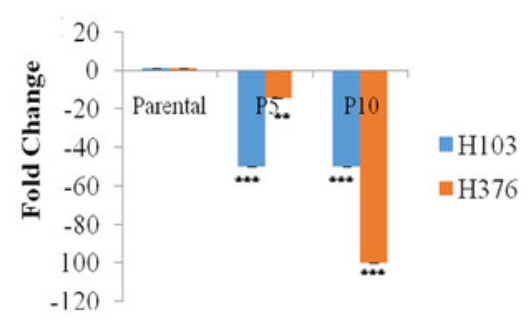

$\mathbf{E}$

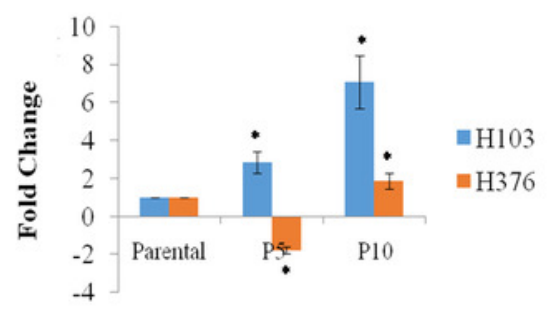




\section{Figure 4}

Figure 4 - Expression of Pluripotent Marker

(A) REP-H103 expressed markers common to pluripotent cells including OCT4, SOX2, NANOG and TRA-1-60. (B) Pluripotency markers (OCT4, SOX2, NANOG and TRA-1-60) were not expressed in parental cancer cell.

A
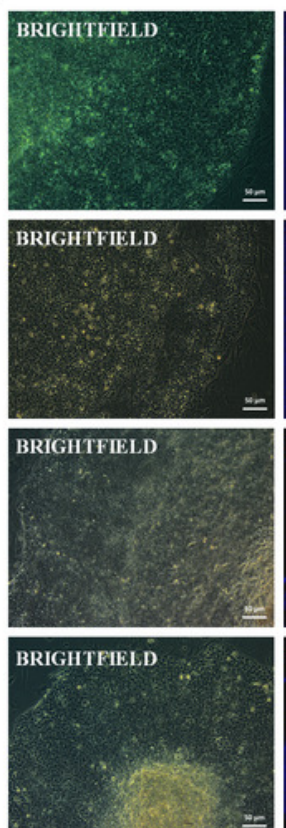

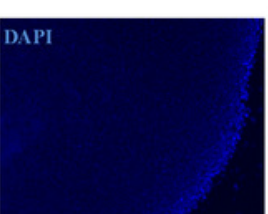

DAPI

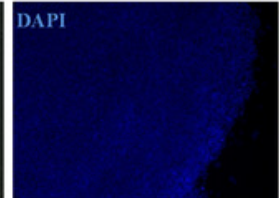

.

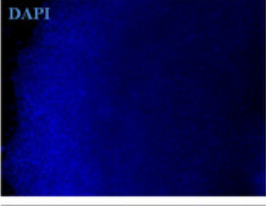

DAPI

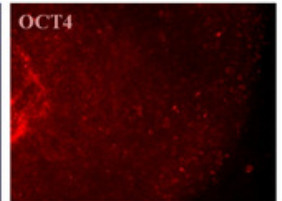

SOX2
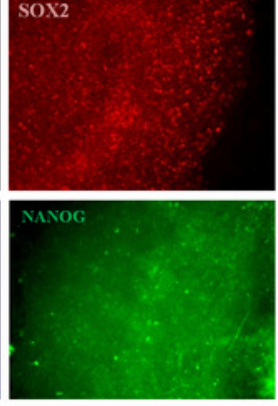

TRA - 1 - 60

B
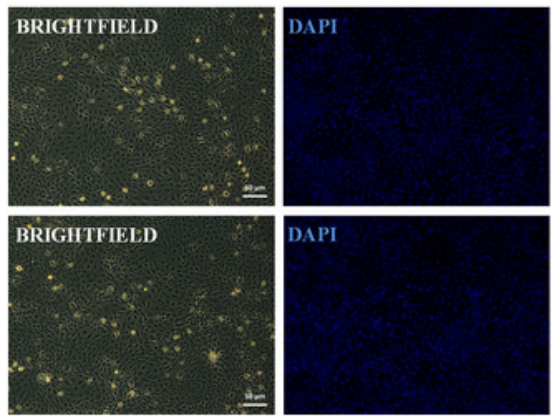

DAPI
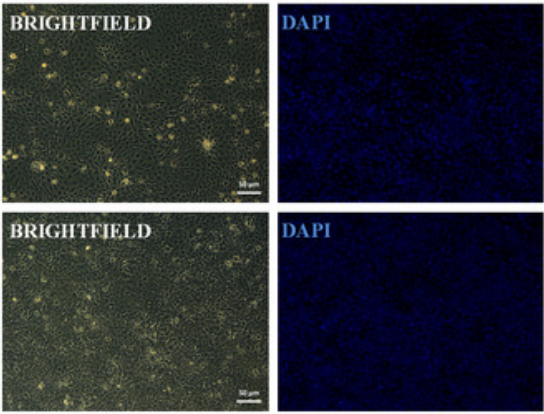

BRIGHTFIELD

DAPI

2. 스.

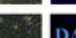

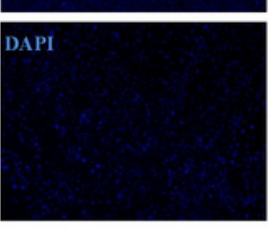

OCT4

SOX2
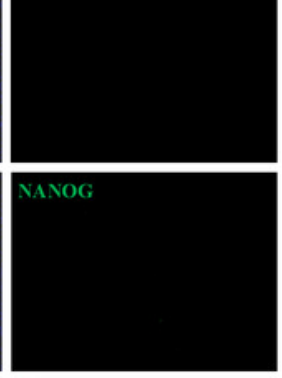

TRA - 1 - 60 


\section{Figure 5}

Fig 5 - Embryoid bodies (EBs) formation and immunofluorescence staining

Representative images of (A-i) parental H103-EB cells and (A-ii) reprogrammed H103-EB. The better showed compact structure and round borders-EB exhibited by reprogrammed H103-EB which is lack in parental H103-EB structure (Red arrow). Nikon inverted microscope, original magnification:10x. Immunostaining analysis indicates the presence of three germ layers of (B-i) ectoderm - OTX2/SOX1, (B-ii) endoderm - GATA4/SOX17 and (B-iii) mesoderm BRACHYURY.

A

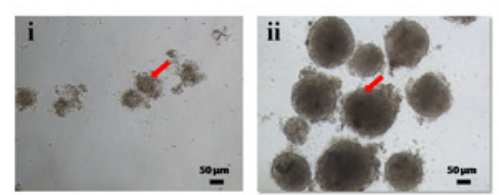

B
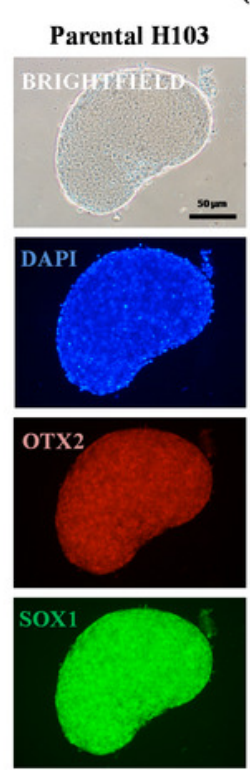

(i)

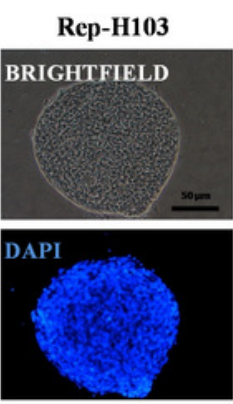

OTX2 (ii)
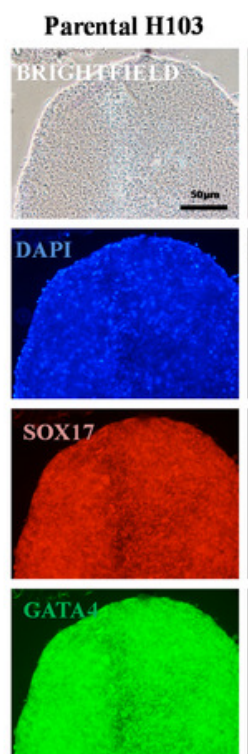

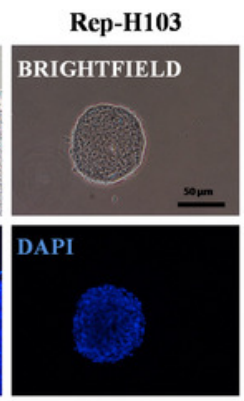

SOX17

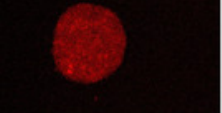

GATA4 (iii)

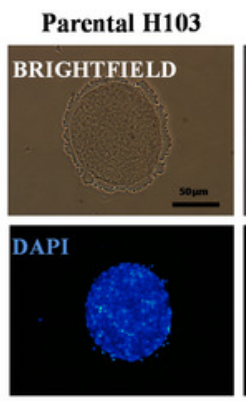

BRACHYURY

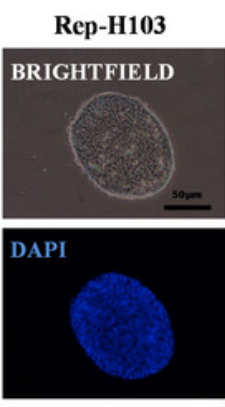

BRACHYURY

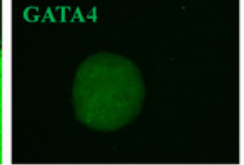

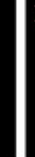




\section{Figure 6}

Fig 6 - Directed differentiations into Osteocytes and Adipocytes

(A-i) Control parental H103 cells showing negative for the Alizarin red staining (A-ii) $\mathrm{H} 103$ IPSCs like cells showing positive for the Alizarin red staining indicating calcium deposits (Red arrow). (A-iii) Negative staining for Oil-Red-O in control parental H103 cells. (A-iv) Adipogenesis induced lipid droplets observed red in colour stain after Oil-O-Red staining. (Red arrow) indicates the tiny lipid droplets. Nikon inverted microscope, original magnification: (A-i \& ii) 10x, (A-iii \& iv) 40x.

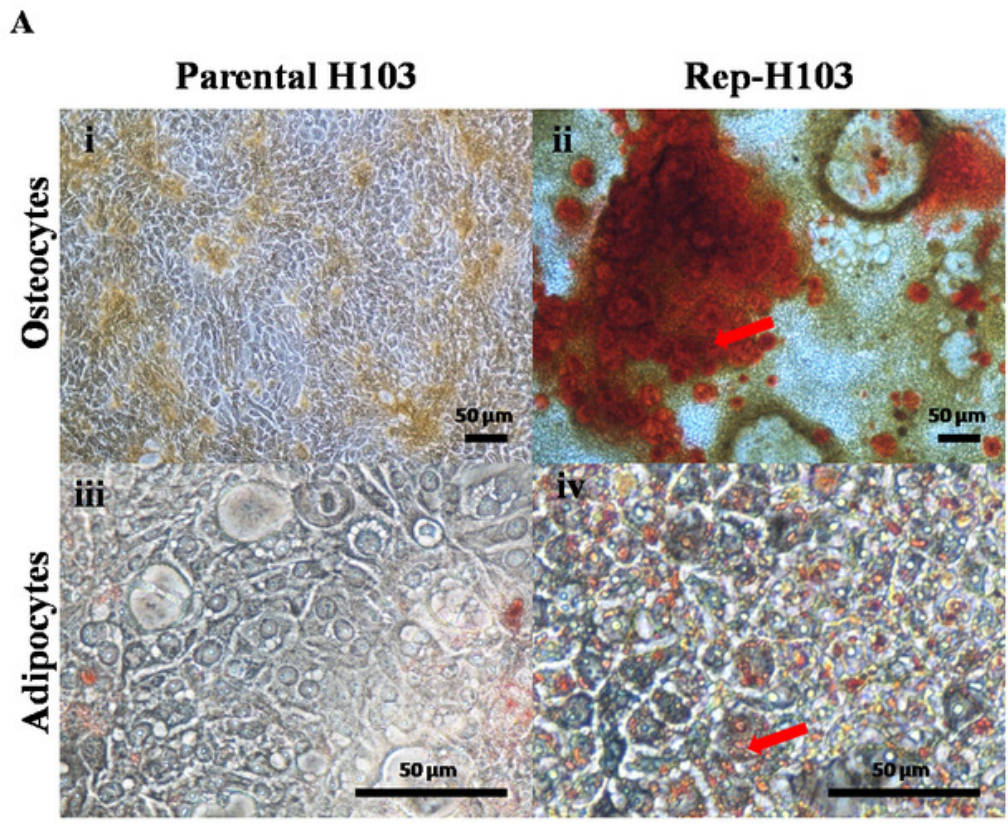

